\title{
Guidelines and the Killer Bs
}

\author{
Derek Richards \\ Editor, Evidence-based Dentistry
}

Evidence-Based Dentistry (2006) 7, 1-2. doi:10.1038/sj.ebd.6400381
We have recently seen the launch of a major new dental guideline from the Scottish Intercollegiate Guidelines Network (SIGN), which we have summarised in this issue. The development of clinical guidelines in healthcare is an expanding industry. Hibble et $a l^{1}$ found 855 different guidelines in general medical practices in Cambridge and Huntingdon Health Authority. There were 243 single-page and 195 two-page guidelines, representing a pile $68 \mathrm{~cm}$ high and weighing $28 \mathrm{~kg}$ ! The number and volume in dentistry is similarly sizeable, as a visit to the FDI World Dental Federation guideline database (www.fdiworldental.org/resources/ 2_oguidelines.html) will confirm.

The definition of a guideline has changed little since Field and Lohr, ${ }^{2}$ upon which SIGN bases its definition:

"systematically developed statements to assist practitioner and patient decisions about appropriate health care for specific clinical circumstances. Guidelines provide recommendations for effective practice in the management of clinical conditions where variations in practice are known to occur and where effective care may not be delivered uniformly (throughout Scotland)."

As clinicians we have seen and received many guidelines but do they change practice?

A recent systematic review, ${ }^{3}$ conducted for the UK National Health Service Research and Development Health Technology Assessment (HTA) programme, identified 235 studies which met the inclusion criteria and reported 309 comparisons of dissemination and implementation methods. The majority (86\%) observed improvements in care but there was considerable variation in the observed effects both within and across interventions. In a later editorial, Grimshaw and Eccles ${ }^{4}$ summarised the main findings:

- there was a median 10\% improvement across studies, suggesting that it is possi- ble to change healthcare-provider behaviour and improve quality of care;

- most dissemination and implementation strategies resulted in small to moderate improvements in care; and

- multifaceted interventions did not appear to be more effective than single interventions.

In their conclusions, Grimshaw and Eccles highlighted the imperfect evidence-base that supports decisions about guideline dissemination and implementation strategies, noting that guidelines are also likely to be efficient under different circumstances. They emphasised the need to develop and validate a coherent theoretical framework of health-professional and organisational behaviour, and of behaviour change, to better inform the choice of interventions in research and service settings, and to estimate the efficiency of dissemination and implementation strategies in the presence of different barriers and effect modifiers. Despite this, it is worth noting that, although a $10 \%$ improvement might be considered modest from a population-health perspective, it is likely to be clinically important. Few dental papers are included in the Grimshaw review; ${ }^{3}$ in this issue of Evidence-based Dentistry we take a look at one recent trial $^{5}$ evaluating implementation of a guideline on lower third molar management.

SIGN (www.sign.ac.uk) was formed in 1993, making it one of the oldest evidence-based guideline organisations. The network's objective is to improve the quality of healthcare for patients in Scotland by reducing variation in practice and outcome, through the development and dissemination of national clinical guidelines containing recommendations for effective practice based on current evidence. SIGN membership embraces all the medical specialties, nursing, pharmacy, dentistry, professions allied to medicine, patients, health service managers, social services and researchers.

To date, SIGN has developed a programme of 113 evidence-based clinical guidelines which are either published, in development or under review, and which encompass a wide range of topics. The latest SIGN dental guideline is SIGN 83: Prevention and Management of Dental Decay in the Pre-school Child. ${ }^{6}$ This new guideline joins SIGN 47: Preventing Dental Caries in Children at High Caries Risk: Targeted Prevention of Dental Caries in the Permanent Teeth of 6-16 Year Olds Presenting for Dental Care ${ }^{7}$ and SIGN 43: Management of Unerupted and Impacted Third Molar Teeth. ${ }^{8}$

Sign's guidelines have a robust methodology and fulfil most, if not all, of the AGREE (Appraisal of Guidelines Research and Evaluation) collaboration criteria, details of which can be found on their website (www.agreecollaboration.org) together with their extensive appraisal instrument. The AGREE instrument consists of 23 key items organised in six domains. Each domain is intended to capture a separate dimension of guideline quality:

- Scope and purpose (items 1-3) is concerned with the overall aim of the guideline, specific clinical questions and target patient population.

- Stakeholder involvement (items 4-7) focuses on the extent to which the guideline represents the views of its intended users.

- Rigour of development (items 8-14) relates to the process used to gather and synthesise the evidence, and the methods to formulate and update the recommendations.

- Clarity and presentation (items 15-18) deals with language and format of the guideline.

- Applicability (items 19-21) pertains to likely organisational, behavioural and 
cost implications of applying the guideline.

- Editorial independence (items 22-23) is concerned with the independence of recommendations and acknowledgement of possible conflicts of interest from the guideline development group.

Although use of the AGREE appraisal tool for guidelines is a council of perfection, for the average practitioner there are two main elements to consider when assessing guidelines. These are the evidence summary and the instructions or advice for applying it to our patients. When considering whether a guideline is valid or not, ask:

1. Did the developers carry out a comprehensive, reproducible literature review within the last 12 months?

2. Is each of its recommendations tagged by the level of evidence upon which it is based and also linked to a specific citation?

In terms of applicability of the guideline this depends on the extent to which it is in harmony with local or patientspecific factors which Sackett ${ }^{9}$ refers to as the "killer Bs". These are

- Burden. Is the burden of illness or frequency in our community too low to warrant implementation?
- Beliefs. Are the beliefs of the individual patients or communities about the value of the interventions or their consequences incompatible with the guideline? - Bargain. Would the opportunity costs of implementing this guideline constitute a bad bargain in the use of our energy or our communities' resources?

- Barriers. Are the Barriers (geographical, organisational, traditional, authoritarian, legal or behavioural) so high that it is not worth trying to overcome them?

The majority of the interventions outlined in SIGN 83 are relatively low-cost (Bargain). Although there may be some challenges in terms of local beliefs and barriers these are probably less challenging than the burden of disease, which is significant: $49 \%$ of 5 -year-old children have obvious signs of decay. ${ }^{10}$ This burden of disease means that, on average, almost $50 \%$ of 5 -year-old children across Scotland have 3.86-5.45 teeth affected by dental disease, ${ }^{10}$ a challenge for the profession and the implementation of this guideline.

1. Hibble A, Kanka D, Pencheon D, Pooles F. Guidelines in general practice: the new Tower of Babel? BMJ 1998; 317:862-863.

2. Field MJ, Lohr KN (eds). Clinical Practice Guidelines: Directions for a New Program,
Washington, DC: Institute of Medicine, National Academy Press; 1990.

3. Grimshaw JM, Thomas RE, MacLennan G, et al Effectiveness and efficiency of guideline dissemination and implementation strategies. Health Technol Assess 2004; 8(6).

4. Grimshaw JM, Eccles MP. Is evidence-based implementation of evidence-based care possible? Med J Aust 2004; 180(Suppl):S50-S51.

5. van der Sanden WJM, Mettes DG, Plasschaert AJM, Grol RPTM, Mulder J, Verdonschot EH. Effectiveness of clinical practice guideline implementation on lower third molar management in improving clinical decisionmaking: a randomised controlled trial. Eur J Ora Sci 2005; 113:349-354.

6. Scottish Intercollegiate Guidelines Network. Prevention and Management of Dental Decay in the Pre-school Child. A National Clinical Guideline. No. 83. Edinburgh: Scottish Intercollegiate Guidelines Network; 2005.

7. Scottish Intercollegiate Guidelines Network. Preventing Dental Caries in Children at High Caries Risk: Targeted Prevention of Dental Caries in the Permanent Teeth of 6-16 Year Olds Presenting for Dental Care. A National Clinical Guideline. No. 47. Edinburgh: Scottish Intercollegiate Guidelines Network; 2000.

8. Scottish Intercollegiate Guidelines Network. Management of Unerupted and Impacted Third Molar Teeth. A National Clinical Guideline. No. 43. Edinburgh: Scottish Intercollegiate Guidelines Network; 2000

9. Sackett DL, Straus SE, Richardson WS, Rosenberg W, Haynes RB. Evidence-based Medicine: How to Practice and Teach EBM, Edinburgh: Churchill Livingstone; 2000.

10. Merrett MCW, Goold S, Jones CM, et al. National Dental Inspection Programme of Scotland Report of the 2004 Survey of P1 Children. Dundee: Scottish Dental Epidemiological Co-ordinating Committee; 2004. 